\title{
A Comparative Study on Temporal Mobile Access Pattern Mining Methods
}

\author{
Hanan Fahmy \\ Information System Dep, \\ Faculty of Computer and Information \\ Systems, Helwan University, \\ Cairo, Egypt
}

\author{
Maha A.Hana \\ Information System Dep, \\ Faculty of Computer and Information \\ Systems, Helwan University, \\ Cairo, Egypt
}

\author{
Yahia K. Helmy \\ Information System Dep, \\ Faculty of Computer and Information \\ Systems, Helwan University, \\ Cairo, Egypt
}

\begin{abstract}
Mobile users behavior patterns is one of the most critical issues that need to be explored in mobile agent systems. Recently the algorithms of discovering frequent mobile user's behavior patterns have been studied extensively. Existing mining methods have proposed frequent mobile user's behavior patterns statistically based on requested services and location information. Therefore, other studies considered that the mobile user's dynamic behavior patterns are usually associated with temporal access patterns. In this paper, temporal mobile access pattern methods are studied and compared in terms of complexity and accuracy. The advantages and disadvantages of these methods will be summarized as well.
\end{abstract}

Keywords- mobile mining; temporal data mining; mobile services; access pattern.

\section{INTRODUCTION}

The mobile industry has experienced significant growth during the past two decades. Practically the fast expansibility of ubiquitous mobile [1] technology has created a numberless opportunity to gather and extract information from mobile agent systems. Recently a critical problem was exist which is how services and access methods can be provided to mobile users. Mobile services are by definition consumed through a mobile handset, which is defined to mean a pocket-sized device with at least cellular connectivity capabilities. In the above mentioned definition of the mobile industry, network vendors, handset vendors, network operators, virtual network operators, service providers, content aggregators, third party software developers and emerging mobile Internet players are all therefore part of the mobile communications industry [2].

In the ubiquitous mobile computing environments, the mobile users may request diverse kinds of services and applications by mobile devices from arbitrary locations at any time via on networks [3]. Obviously, the behavior pattern, in which the location and the service are inherently coexistent with temporal associated, of mobile users becomes more complex than that of the traditional mobile agent systems. To assist the user get interested information on time is one of the promising applications, especially in mobile agent systems. Wherefore, effective modeling of mobile user behavior patterns is becoming very important. As effective modeling the behavior patterns of users in mobile agent systems benefits not only the users in smart access by caching or pre-fetching [4] [5] but also the mobile service providers to enhance their services and provide new services that may attract more subscribers [6].

There are existed studies that considered only one of the characteristics, i.e. location associated with requested services. Obviously, both movement and location or service requested with the temporal association rule should be considered simultaneously in order to discover complete information of user behavior patterns when the user request services. As a result, recently there are studies exist that discover the mobile user's interesting movement behavior patterns by temporal mobile access patterns in which this paper considered. These methods are Temporal Mobile access pattern (TMAP) [7] and Temporal mobile sequential pattern (TMSP) [8] as they have advantages and disadvantages of their own.

This paper aims to introduce a comparative study of the tow different methods of discovering temporal mobile behavior as these methods are studied respectively and compared in terms of complexity and accuracy. Furthermore, we explore the advantages and disadvantages of each of them.

The remainder of this paper is organized as follows. In Section 2, we gave a brief definition of mobile service. In Section 3, we briefly define the temporal data mining. Section 4 describes the comparative temporal mining methods in details with introducing the strength and weakness points of each one. Finally, the conclusion is given in Section 5.

\section{MobILE SERVICES}

Mobile services differ from traditional services in their ability to provide service offerings regardless of temporal and spatial constraints. They are also different from traditional interpersonal services that deliver face-to-face, or from other types of e-services, such as wireless online services, where the service delivery linked to a specific fixed local area network or specific location. Mobile services have some special characteristics in comparison to other types of services [9].

The key differences related to spatial and temporal components of service usage [10]. For example, if one wants to meet a bank teller, she has to visit the bank location at a certain appointment time. These restrictions are present to some extent even with many electronic services. Even though online banking over a DSL Internet connection. Even though online banking services are available 24 hours a day thus overcoming 
the problem of temporal availability, a fixed location is still needed for the DSL line, which is a spatial restriction. Mobile services, used with handheld mobile devices, overcome both spatial and temporal constraints [2] [9] an additional unique dimension of mobile services is the potential for individual personalization of service offerings.

Mobile handsets are multi-purpose private computers. This provides an attractive setting for electronic service delivery. Evaluation of mobile services as perceived by end-users is therefore challenging. Some papers claim that the opportunity cost of time is what matters [11]. Some analyses also recognize the dimension of level of effort in using the service [12].

\section{TEMPORAL DATA MINING}

Data mining is defined as the process of nontrivial extraction of previously unknown and useful knowledge from data. It discovers patterns hidden in data and associations between the patterns. Temporal data mining deals with the problem of mining patterns from temporal data, which can be either symbolic sequences or numerical time series [13]. Temporal data mining has the capability to look for interesting correlations or rules in large sets of temporal data.

The definition of temporal data mining presented in [14] is as the following. Temporal Data Mining is a single step in the process of Knowledge Discovery (KD) in temporal databases that enumerates structures (temporal patterns or models) over the temporal data, and any algorithm that enumerates temporal patterns from, or fits models to, temporal data is a temporal data mining algorithm.

Currently, temporal data mining is a fast expanding field with many research results reported. As a result, many new temporal data mining analysis methods or prototypes developed recently [15]. Two factors contribute to the popularity of temporal data mining. The first factor is an increase in the volume of temporal data stored, as many realworld applications deal with huge amount of temporal data. The second factor is the mounting recognition in the value of temporal data [16].

In many application domains, temporal data are now being viewed as invaluable assets from which hidden knowledge can be derived, to help understand the past and/or plan for the future [17].

\section{TEMPORAL Mining MEthods}

In This section, we describe the temporal mobile access pattern mining methods, which are T-MAP mine and TMSP mine. These mining algorithms will be described in details respectively with their mining mechanisms followed by expressing the strength and weakness points of each mining method.

\section{A. TMAP Mine}

Temporal Mobile Access Patterns is a data mining method to discover mobile user's temporal behavior patterns associated with location and request services by using temporal association rule. Furthermore, it used data structure with temporal mobile access patterns called T-Map-Tree [7]. As shown in Fig 1 the workflow of TMAP mining mechanism contains two phases, which are data integration phase and mining phase. Data integration phase, is to collect and integrate users' $\log$ s into one dataset. Mining phase is to discover the frequently temporal mobile access patterns (T-Map) from the integrated log dataset [18].

TMAP use predefined timestamps by setting the time interval every four hours then they integrate the mobile user's current location information and service request into the integrated log as time goes by according to TMAP predefined time interval. The TMAP mine is consisted of a header table and aggregating the access patterns into the memory in a compact form. The head table are stored the frequently occurrence of mobile access patterns in the order of descending sequence access patterns. TMAP method needs one physical database scan when constructing of the header table. The database scan is to find all frequent mobile access patterns. Then, the frequent mobile access pattern is inserted into the header table in decreasing order of their sequence access pattern After the scan of the database, the set of frequent access patterns is sorted in the order of descending sequence data then the TMAP structure is constructed.

TMAP is conducted to efficiently find the mobile users' temporal mobile access pattern in distinct time interval, which is helpful to provide real-time customized personal service. However, TMAP method lacks of flexibilities because for each time interval segmentation the start time and end time should be determined and not every data is suitable for same cutting method. Finding the best segmentation in not easy in advance, the predication rate will be influenced by the segmenting point of time interval.

Although TMAP method is a fast method due to using the compact data structure form, but the log data sets consume much memory when the information data sets are storing into the memory. TMAP method works especially well for contextawareness data sets in mobile agent systems however, TMAP is not applied on real datasets, and its performance is not evaluated.

\section{B. TMSP Mine}

Temporal Mobile Sequential Patterns is a data mining method for discovering the Temporal Mobile Sequential Patterns (TMSPs) of mobile users in Location Based Services (LBS) environments. Furthermore, it uses location prediction strategies to predict the next movement of mobile users by utilizing the discovered TMSPs [8]. As shown in Fig 2 the workflow of TMSP mining mechanism contains three phases: segmentation of mobile transactions, discovery of TMSPs, and prediction user's behaviors. The segmentation of mobile transactions phase use the statistical method to decide a suitable count of time segmenting points and base on it to obtain the most suitable position of time segmenting points by genetic algorithm (GA). In the discovery of TMSPs phase, the mobile logs of users are analyzed by a data mining algorithm to obtain the TMSPs for each time interval. Furthermore, the TMSPs are used to predict the next location and service of mobile users. In prediction phase, the most suitable pattern is picked up by the historical transaction log, moving path, and current time interval to provide LBS like in advance preparing the service which user might request in advance, and 
recommending related services, and user can query related service as well.

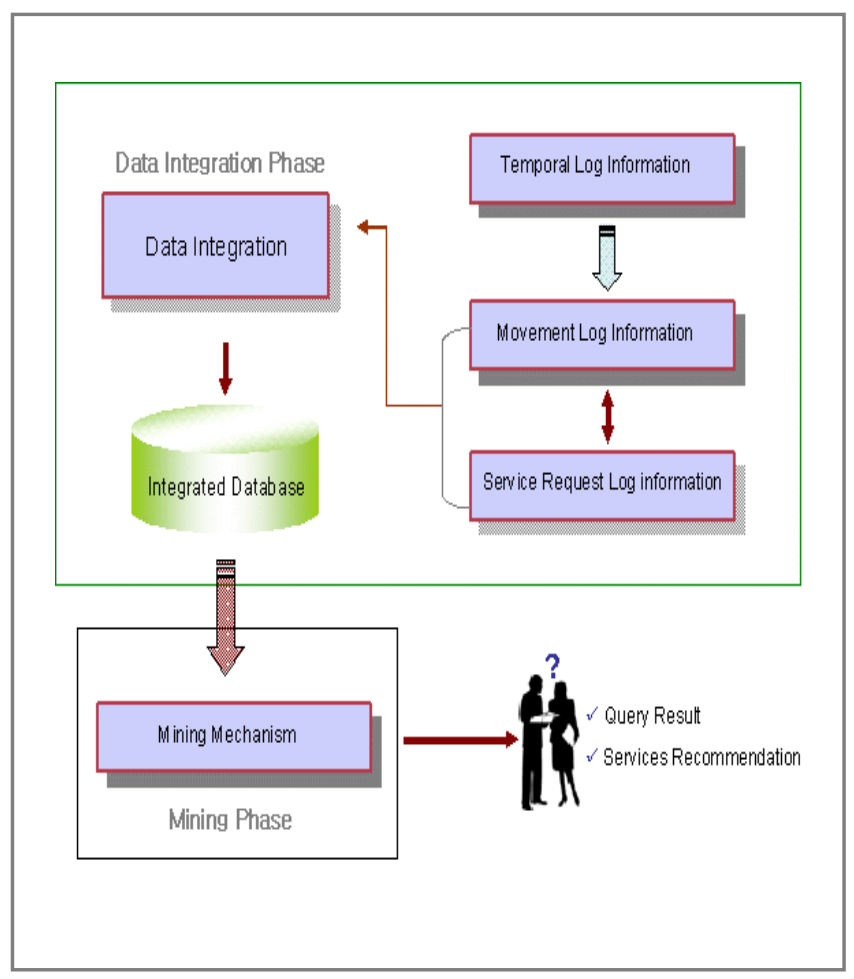

Figure 1. Work mechanisms for Temporal Mobile Access Pattern[18]

TMSP refer to TMAP-mine algorithm [8] without using time interval but using genetic algorithm to discover the most suitable time intervals by obtaining the most suitable positions of time segmenting point. TMSP-Mine algorithm determines the large transactions for each time interval and cell. TMSPMine algorithm uses the mapping table to transform the mobile transaction sequences into maximal large transaction sequences. By this step, TMSP gains advantage that can reduce the services of transactions that less than minimal support.

TMSP-Mine Algorithm utilizes TMSP-Tree, which is a two-level tree. The TMSP-Tree is well structured when comparing two patterns that have the same first and last transaction, and to generate candidate mobile sequential efficiently. This action is repeated more than one time until no candidate patterns can be generated which consume much time and memory space. TMSP use three prediction strategies for predicting behaviors of users by selecting the TMSPs based on the corresponding time interval; or with the longest length of fitting mobile user's previous location, service request, and moving path. If there are many TMSPs with the same length, TMSP is selected with the maximal support.

Although TMSP- mine method used for discovering the Temporal Mobile Sequential Patterns (TMSPs) of mobile users but the time segment is generated by searching into the time interval of the transaction $\log$ file that consume much time of searching; however it is no absolute assurance that a genetic algorithm will find a global optimum [19]. In addition, it store record for location without service in the dataset followed by record for location with service, which waste much memory space.
The genetic algorithm cannot assure constant optimization response times. Even more, the difference between the shortest and the longest optimization response time is much larger than with conventional gradient methods. This unfortunate genetic algorithm property limits the genetic algorithms' use in real time applications [20].

Figure 2. Work mechanisms for Temporal Mobile Sequential Pattern[8]

$$
\text { V. CONCLUSION }
$$

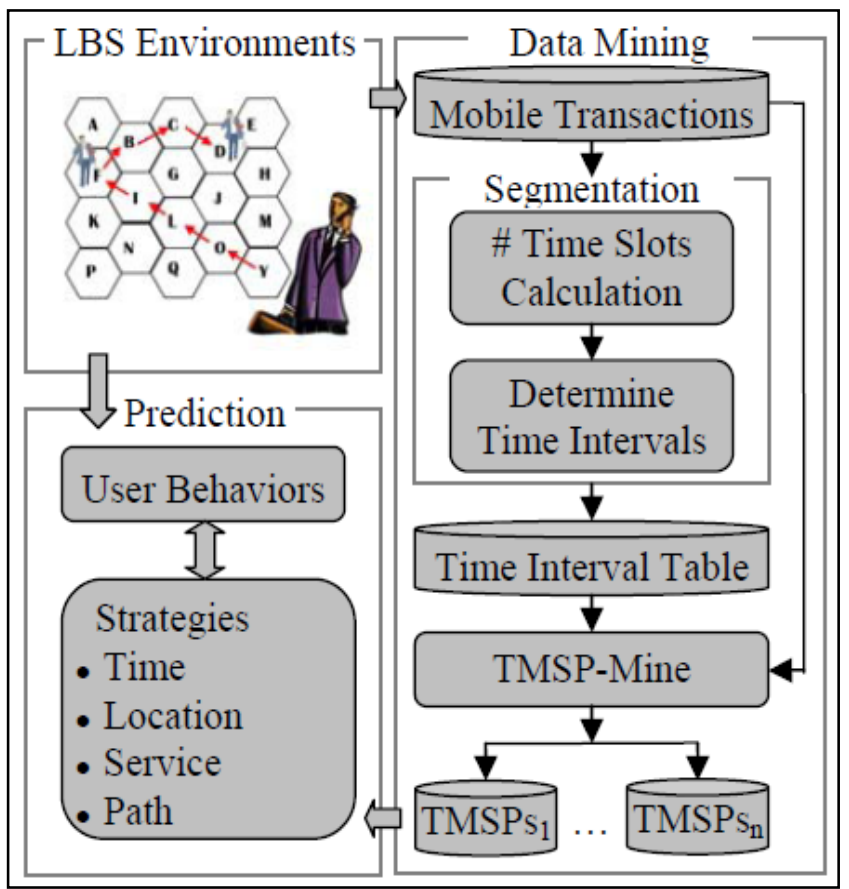

In this paper, we introduce a comparative study on temporal mobile behavior patterns after studying the existing mining methods. The two different temporal mobile access mining methods, which are TMAP and TMSP, are studied and compared in terms of complexity and accuracy. Our study shows that each one of them has their own advantages and disadvantages. Although the two algorithms used to predict the mobile behavior pattern in terms of service and location without concerning the dynamic time interval based of the response time of mobile service providers.

As a result, it remains an open question how to construct the best association rule based on sequential and temporal association rules for mobile users' access pattern for best recommend the next service that the mobile user will request in a dynamic timely manager according to the average response time of the mobile service providers. References

[1] Dan Lim,"Ubiquitous mobile Computing: UMC's model and success", Educational Technology \& society 2(4) ISSN 1436-4522,1999.

[2] Hannu T. Verkasalo," Handset-Based Analysis of Mobile Service Usage",Phd Helsinki University of Technology, Faculty of Electronics, Communications and Automation, Department of Communications and Networking,2009. 
[3] Yu ning, Hongbin Yang, "Sequence Mining for user behavior patterns in mobile Commerce", International Conference on Management of eCommerce and e-Government, 978-0-7695-3366-7/08 \$25.00, IEEE 2008.

[4] J.L.Chen," Resource allocation for cellular data services using multiagent schemes", IEEE Trans. Syst. Man Cybern. 31 (6) (2001) 864869,2001 .

[5] Vincent S. Tseng and W. C. Lin.,"Mining Sequential Mobile Access Patterns Efficiently in Mobile Web Systems", Proceeding of International Conference on Advanced Information Networking and Applications, pages 867-871, Taipei, Taiwan, March 2005.

[6] C.Y Chang, M.S Chen, "Integrating web caching and web prefetching in client-side proxies", in: Proceedings of the ACM 11th International conference Information and knowledge Management, 2002.

[7] S. C. Lee, J. Paik, J. Ok, I. Song, and U. M. Kim, "Efficient Mining of User Behaviors by Temporal Mobile Access Patterns". International Journal of Computer Science Security, Vol. 7, No. 2, pages 285-291, February 2007.

[8] Vincent S. Tseng, Eric Hsueh-Chan Lu, Cheng-Hsien Huang, "Mining Temporal Mobile Sequential Patterns in Location-Based Service Environments" , 978-1-4244-1890-9/07, IEEE 2007.

[9] Heinonen, K., Pura, M. , "Classifying Mobile Services", Proceedings of Helsinki Mobility Roundtable. Sprouts: Working Papers on Information Systems, 6(42). http://sprouts.aisnet.org/6-42, 2006.

[10] J. F. Roddick, K. Hornsby and M. Spiliopoulou, "Temporal Spatial and Spatio-Temporal data mining and knowledge discovery research Bibliography", http://kdm.first.flinders.edu.au/IDM/STDMBib.html.

[11] Goolsbee, A. \& Klenow, PJ., "Valuing Consumer Products by the Time Spent Using Them: An Application to the Internet", Presented at the AEA Session on "The Roots of Innovation," Boston, Massachusetts,
January 8, 2006.

[12] Pohjola, OP \& Kilkki, K., "Value-based methodology to analyze communication services", Presented at Conference on Telecommunication Techno-Economics (CTTE) 2006.

[13] Dr. Naveeta Mehta et al. , " Temporal Sequential Pattern In Data Mining Tasks", International Journal on Computer Science and Engineering (IJCSE), 2011.

[14] Lin, W., Orgun, M. A. \& Williams, G. J. ,"An overview of temporal data mining", in S. J. Simoff, G. J. Williams \& M. Hegland, eds, Proceedings of the 1st Australasian Data Mining Workshop (ADM'02)', Sydney, Australia, pp. 83-89,2002.

[15] Srikant R, Agrawal R: "Mining Sequential Patterns: Generalizations and Performance Improvements", in Int'l Conf Extending Database Technology. Springer 1996.

[16] Edi Winarko," The Discovery and Retrieval of Temporal Rules in Interval Sequence Data", Phd Flinders University, Faculty of Science and Engineering,2007.

[17] Chen, X. \& Petrounias, I. ," A framework for temporal data mining", in 'Proceedings of the 9th International Conference on Database and Expert Systems Applications (DEXA'98)', Vienna, Austria, pp. 796$805,1998$.

[18] SeungCheol Lee, J. paik, J. Ok, I, Song and Ung Mo Kim, "Extracting Temporal Behavior Patterns of Mobile User", 978-0-7695-3322-3/08, IEEE 2008.

[19] William Sayers, "Genetic Algorithms and Neural Networks", Faculty of Advanced Technology, 05025397, milestone 3, Page 25-26,2009.

[20] Marczyk, A., "Genetic algorithms and evolutionary computatio"., http://www.talkorigins.com/faqs/genalg/genalg.htm, from Retrieved April13,2009. 